\title{
Novel Ways to Look for the Critical Point and New Phases in Relativistic Heavy lon Collisions
}

\author{
Masayuki Asakawa*t \\ Department of Physics, Osaka University, Toyonaka 560-0043 Japan \\ E-mail: yuki@phys.sci.osaka-u.ac.jp
}

We discuss two novel ways to look for the critical point and new phases in relativistic heavy ion collisions. The two quantities are not subject to final state interactions unlike other commonly proposed signals.

5th International Workshop on Critical Point and Onset of Deconfinement - CPOD 2009,

June 08 - 122009

Brookhaven National Laboratory, Long Island, New York, USA

\footnotetext{
* Speaker.

${ }^{\dagger}$ This work is supported in part by a Grant-in-Aid for Scientific Research by Monbu-Kagakusyo of Japan (No. 20540268).
} 


\section{Third Moments of Conserved Charges}

Quantum chromodynamics (QCD) is believed to have a rich phase structure in the temperature $(T)$ and baryon chemical potential $\left(\mu_{\mathrm{B}}\right)$ plane. Lattice QCD calculations indicate that the chiral and deconfinement phase transitions are a smooth crossover on the temperature axis [1], while various models predict that the phase transition becomes of first order at high density [2]. The existence of the QCD critical point is thus expected. To map these components of the phase diagram on the $T-\mu_{\mathrm{B}}$ plane is one of the most challenging and stimulating subjects which may be achieved by relativistic heavy ion collisions.

Various observables have been proposed for this purpose [3, 4, 5, 6]. Most scenarios suggested so far are concerned with fluctuations, such as those of conserved charges, momentum distributions, slope parameters, and so forth. For example, fluctuations of conserved charges behave differently between the hadronic and quark-gluon plasma phases, and may be used as an indicator of the realization of the phase transition [4, 5]. The singularity at the critical point, at which the transition is of second order, may also cause enhancements of fluctuations if fireballs created by heavy ion collisions pass near the critical point during the time evolution [3, 6]. Because of finite size effects and critical slowing down, however, such singularities are blurred and its experimental conformation may not be possible [8, 9]. In fact, so far no clear evidence for the critical point has been detected in event-by-event analyses. Approaches to use higher order moments for this purpose have been also suggested recently [7] and experimental attempts to measure those higher order moments were reported. Almost all previous studies, however, focus on the absolute value, especially the enhancement, of each observable around the phase boundary.

Here we propose to employ signs of third moments of conserved charges around the averages, which we call, for simplicity, the third moments in the following, to infer the states created by heavy ion collisions. In particular, we consider third moments of conserved quantities, the net baryon and electric charge numbers, and the energy,

$$
m_{3}(c c c) \equiv \frac{\left\langle\left(\delta N_{c}\right)^{3}\right\rangle}{V T^{2}}, \quad m_{3}(\mathrm{EEE}) \equiv \frac{\left\langle(\delta E)^{3}\right\rangle}{V T^{5}},
$$

where $N_{c}$ with $c=\mathrm{B}, \mathrm{Q}$ represent the net baryon and electric charge numbers in a subvolume $V$, respectively, $E$ denotes the total energy in $V, \delta N_{c}=N_{c}-\left\langle N_{c}\right\rangle$, and $\delta E=E-\langle E\rangle$. We also make use of the mixed moments defined as follows:

$$
m_{3}(c c \mathrm{E}) \equiv \frac{\left\langle\left(\delta N_{c}\right)^{2} \delta E\right\rangle}{V T^{3}}, \quad m_{3}(c \mathrm{EE}) \equiv \frac{\left\langle\delta N_{c}(\delta E)^{2}\right\rangle}{V T^{4}} .
$$

To understand the behaviors of these moments around the QCD phase boundary, we first notice that the moments Eqs. (1.1) and (1.2) are related to third derivatives of the thermodynamic potential per unit volume, $\omega$, with respect to the corresponding chemical potentials and $T$. The simplest example is $m_{3}(\mathrm{BBB})$, which is given by

$$
m_{3}(\mathrm{BBB})=-\frac{\partial^{3} \omega}{\partial \mu_{\mathrm{B}}^{3}}=\frac{\partial \chi_{\mathrm{B}}}{\partial \mu_{\mathrm{B}}},
$$

where the baryon number susceptibility, $\chi_{\mathrm{B}}$, is defined as

$$
\chi_{\mathrm{B}}=-\frac{\partial^{2} \omega}{\partial \mu_{\mathrm{B}}^{2}}=\frac{\left\langle\left(\delta N_{\mathrm{B}}\right)^{2}\right\rangle}{V T} .
$$


The baryon number susceptibility $\chi_{\mathrm{B}}$ diverges at the critical point and has a peak structure around there [3, 10]. Since $m_{3}(\mathrm{BBB})$ is given by the $\mu_{\mathrm{B}}$ derivative of $\chi_{\mathrm{B}}$ as in Eq. (1.3), the existence of the peak in $\chi_{\mathrm{B}}$ means that $m_{3}(\mathrm{BBB})$ changes its sign there. Although the precise size and shape of the critical region are not known, various models predict that the peak structure of $\chi_{\mathrm{B}}$ well survives far along the crossover line [2, 10,11] (See, Fig. 1 as a demonstration of this feature in a simple effective model; the details will be explained later). This means that the near (hadron) and far (quark-gluon) sides of the QCD phase boundary can be distinguished by the sign of $m_{3}(\mathrm{BBB})$ over a rather wide range around the critical point. It is this feature that third moments carry more information than fluctuations (second moments); fluctuations are, by definition, positive definite and cannot differentiate the near side from the far side as decisively as the third moments. We note that odd power moments around the averages in general do not vanish except the first order one. As we shall see later, all third moments presented in Eqs. (1.1) and (1.2) are related to derivatives of corresponding susceptibility which diverges at the QCD critical point, and hence change their signs there.

Once the negativeness of third moments is established experimentally, it is direct evidence of two facts: (1) the existence of a peak structure of corresponding susceptibility in the phase diagram of QCD, and (2) the realization of hot matter beyond the peak, i.e. the quark-gluon plasma, in heavy ion collisions. We emphasize that this statement using the signs of third moments is free from any theoretical ambiguities. The experimental measurements of signs of moments also have an advantage compared to their absolute values: it is usually essential to normalize experimentally obtained values by observables proportional to the volume in order to extract physically meaningful quantities [4, 5]. In the measurement of signs, however, normalization is not necessary. It is this feature that our proposal is less subject to experimental and theoretical ambiguities and more robust than previously proposed ones.

Let us now consider the behavior of third moments other than $m_{3}(\mathrm{BBB})$ around the critical point. First, the third moment of the net electric charge $m_{3}(\mathrm{QQQ})$ is calculated to be

$$
m_{3}(\mathrm{QQQ})=-\frac{1}{8} \frac{\partial^{3} \omega}{\partial \mu_{\mathrm{B}}^{3}}-\frac{3}{8} \frac{\partial^{3} \omega}{\partial \mu_{\mathrm{B}}^{2} \mu_{\mathrm{I}}}-\frac{3}{8} \frac{\partial^{3} \omega}{\partial \mu_{\mathrm{B}} \mu_{\mathrm{I}}^{2}}-\frac{1}{8} \frac{\partial^{3} \omega}{\partial \mu_{\mathrm{I}}^{3}},
$$

where $\mu_{\mathrm{Q}}$ represents the chemical potential associated with $N_{\mathrm{Q}}$, i.e. $\partial / \partial \mu_{\mathrm{Q}}=(2 / 3) \partial / \partial \mu_{\mathrm{u}}-$ $(1 / 3) \partial / \partial \mu_{\mathrm{d}}=\left(\partial / \partial \mu_{\mathrm{B}}+\partial / \partial \mu_{\mathrm{I}}\right) / 2$, and the isospin chemical potential is defined as $\mu_{\mathrm{I}}=\left(\mu_{\mathrm{u}}-\right.$ $\left.\mu_{\mathrm{d}}\right) / 2$ with $\mu_{\mathrm{u}, \mathrm{d}}$ being the chemical potentials of the up and down quarks, respectively. In relativistic heavy ion collisions, the effect of isospin symmetry breaking is small. Assuming the isospin symmetry, the second and last terms in Eq. (1.5) vanish and one obtains

$$
m_{3}(\mathrm{QQQ})=\frac{1}{8} \frac{\partial}{\partial \mu_{\mathrm{B}}}\left(\chi_{\mathrm{B}}+3 \chi_{\mathrm{I}}\right)
$$

with the isospin susceptibility $\chi_{\mathrm{I}}=-\partial^{2} \omega / \partial \mu_{\mathrm{I}}^{2}$. Under the isospin symmetry, $\chi_{\mathrm{I}}$ does not diverge at the critical point because the critical fluctuation does not couple to the isospin density [6]. The critical behavior of the term in the parenthesis in Eq. (1.6) in the vicinity of the critical point is thus solely governed by $\chi_{\mathrm{B}}$. Since $m_{3}(\mathrm{QQQ})$ is a $\mu_{\mathrm{B}}$ derivative of this term, a similar behavior as $m_{3}(\mathrm{BBB})$ is expected. 


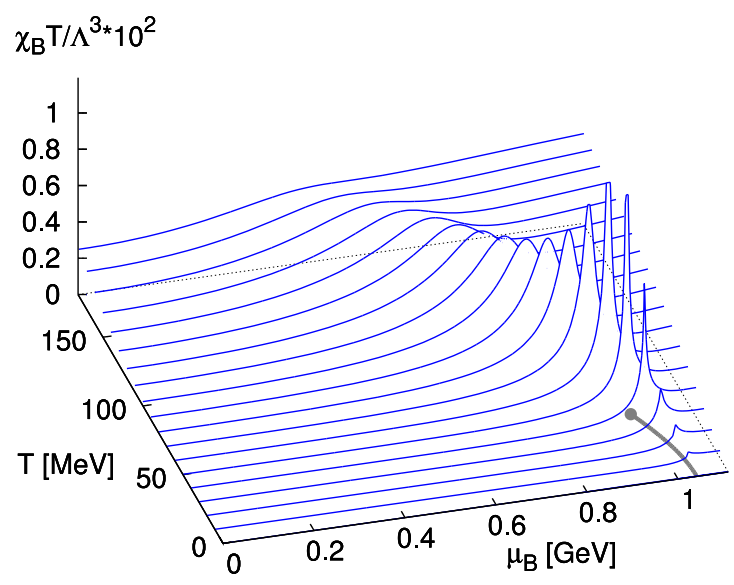

Figure 1: $T$ and $\mu_{\mathrm{B}}$ dependence of the baryon number susceptibility $\chi_{\mathrm{B}}$ multiplied by $T$ in the Nambu-JonaLasinio model. The bold line on the bottom surface shows the first order phase transition line and the point at the end is the critical point.

Next, it can be shown that mixed moments including a single $\mathrm{E}$ are concisely given by

$$
m_{3}(c c \mathrm{E})=\left.\frac{1}{T} \frac{\partial\left(T \chi_{c}\right)}{\partial T}\right|_{\hat{\mu}}
$$

with $c=\mathrm{B}$, Q, where $\chi_{\mathrm{Q}} \equiv-\partial^{2} \omega / \partial \mu_{\mathrm{Q}}^{2}=\left(\chi_{\mathrm{B}}+\chi_{\mathrm{I}}\right) / 4$ is the electric charge susceptibility. The $T$ derivative in Eq. (1.7) is taken along the radial direction from the origin with fixed $\hat{\mu} \equiv \mu_{\mathrm{B}} / T$, i.e. $\partial /\left.\partial T\right|_{\hat{\mu}}=\partial /\left.\partial T\right|_{\mu_{\mathrm{B}}}+\left(\mu_{\mathrm{B}} / T\right) \partial /\left.\partial \mu_{\mathrm{B}}\right|_{T}$. Since $T \chi_{c}$ diverges at the critical point, Eq. (1.7) again leads to a similar behavior of $m_{3}(c c \mathrm{E})$ as the above-mentioned moments.

To argue the behaviors of remaining third moments including two or three E's, it is convenient to first define $C_{\hat{\mu}}=-T\left(\partial^{2} \omega / \partial T^{2}\right)_{\hat{\mu}}=\left\langle(\delta E)^{2}\right\rangle / V T^{2}$. The third moments are then given by

$$
m_{3}(\mathrm{EEE})=\left.\frac{1}{T^{3}} \frac{\partial\left(T^{2} C_{\hat{\mu}}\right)}{\partial T}\right|_{\hat{\mu}}, \quad m_{3}(\mathrm{BEE})=2 m_{3}(\mathrm{QEE})=\frac{1}{T} \frac{\partial C_{\hat{\mu}}}{\partial \mu_{\mathrm{B}}} .
$$

Since $C_{\hat{\mu}}$ is the second derivative of $\omega$ along the radial direction, it diverges at the critical point which belongs to the same universality class as that of the 3D Ising model. Therefore, $m_{3}(\mathrm{EEE})$, $m_{3}(\mathrm{BEE})$, and $m_{3}(\mathrm{QEE})$, all change their signs at the critical point.

While the above arguments, based on the divergence of second derivative of $\omega$, guarantee the appearance of the region with negative third moments in the vicinity of the critical point, they do not tell us anything about the size of these regions in the $T-\mu_{\mathrm{B}}$ plane. In fact, all third moments considered here become positive at sufficiently high $T$ and $\mu_{\mathrm{B}}>0$ where the system approaches a free quark and gluon system. The regions are thus limited more or less near the critical point.

The range of $\mu_{\mathrm{B}} / T$ where lattice simulations are successfully applied is limited to small $\mu_{\mathrm{B}} / T$ with the present algorithms. In particular, thermodynamics around the critical point cannot be analyzed with the Taylor expansion method. In order to evaluate the qualitative behavior of the third moments in such a region, one has to resort to effective models of QCD. To make such an estimate, here we employ the two-flavor Nambu-Jona-Lasinio model [12, 13] with the standard interaction 


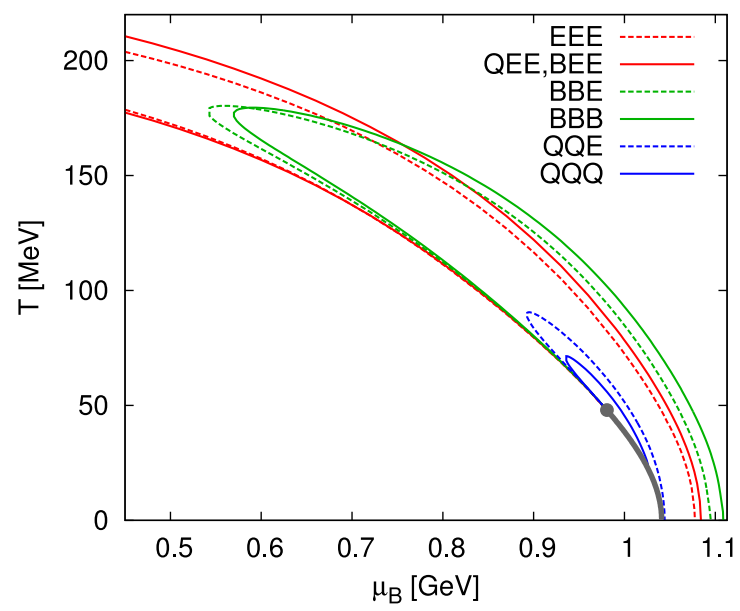

Figure 2: Regions where third moments take negative values in the $T-\mu_{\mathrm{B}}$ plane. The regions are inside the boundaries given by the lines.

$\mathscr{L}_{\text {int }}=G\left\{(\bar{\psi} \psi)^{2}+\left(\bar{\psi} i \gamma_{5} \tau_{i} \psi\right)^{2}\right\}$, where $\psi$ denotes the quark field. For the model parameters, we take the values determined in Ref. [12]; $G=5.5 \mathrm{GeV}^{-2}$, the current quark mass $m=5.5 \mathrm{MeV}$, and the three-momentum cutoff $\Lambda=631 \mathrm{MeV}$. For the isospin symmetric matter, this model gives a first order phase transition at large $\mu_{\mathrm{B}}$, as shown on the bottom surface of Fig. 1 by the bold line. The critical point is at $\left(T, \mu_{\mathrm{B}}\right) \approx(48,980) \mathrm{MeV}$.

In Fig. 1, we also show the $T$ and $\mu_{\mathrm{B}}$ dependence of $T \chi_{\mathrm{B}}$ calculated in the mean-field approximation. One observes that $\chi_{\mathrm{B}}$ diverges at the critical point, and the peak structure well survives along the crossover line up to higher temperatures [10]. The region where each moment becomes negative in the $T-\mu_{\mathrm{B}}$ plane is shown in Fig. 2, One sees that all the moments become negative on the far side of the critical point as it should be, whereas the extent of the region depends on the channel. The figure shows that areas with $m_{3}(\mathrm{BBB})<0$ and $m_{3}(\mathrm{BBE})<0$ extend to much lower $\mu_{\mathrm{B}}$ and much higher $T$ than the critical point. This suggests that even if the critical point is located at high $\mu_{\mathrm{B}}$ the negative third moments can be observed by heavy ion collision experiments. The figure also shows that the areas have considerable thicknesses along the radial direction. Since the system stays near the phase transition line considerably long regardless of the order of the phase transition, first order or crossover, once the state on the far side is created, negative third moments are very likely to be formed and observed. The wide regions of negative moments also indicate that they are hardly affected by critical slowing down during the dynamical evolution of fireballs.

Figure 2 also shows that areas with negative $m_{3}(\mathrm{EEE}), m_{3}(\mathrm{QEE})$ and $m_{3}(\mathrm{BEE})$ are much larger than those of the other moments in the $T$ - $\mu_{\mathrm{B}}$ plane; although not shown in the figure, these areas extend even to the $T$-axis. The behaviors of $m_{3}(\mathrm{EEE})$ and $m_{3}(c \mathrm{EE})$ near the $T$-axis can be checked directly by the lattice simulations. If the range of $T$ satisfying $m_{3}(\mathrm{EEE})<0$ is sufficiently wide at $\mu_{\mathrm{B}}=0$, it is possible that the negative third moments are measured even at the RHIC and LHC energies. Whether the negative moments survive or not in this case depends on the diffusion time of the energy density, in other words the heat conductivity. One can thus use the signs of $m_{3}(\mathrm{EEE})$ and $m_{3}(c \mathrm{EE})$ to estimate the diffusion time of the charges and energy. The third moments $m_{3}(\mathrm{QQQ})$ 
and $m_{3}(\mathrm{QQE})$, on the other hand, become negative only in a small region near the critical point. These behaviors come from the large contribution of $\chi_{\mathrm{I}}$ in Eq. (1.6).

It should be, however, remembered that the results in Figs. 1 and 2, are obtained in an effective model. In particular, the model employed here gives the critical point at relatively low $T$ and high $\mu_{\mathrm{B}}$ [14]. If the critical point is at much lower $\mu_{\mathrm{B}}$, the areas with negative moments in Fig. 2 should also move toward lower $\mu_{\mathrm{B}}$ and higher $T$.

Here we have pointed out that the third moments of conserved charges, the net baryon and electric charge numbers and the energy, carry more information on the state around the QCD phase boundary than usual fluctuation observables. They change signs at the phase boundary corresponding to the existence of the peaks of susceptibilities. If the negative third moments grow at early stage of the time evolution of fireball created in the collisions and if the diffusion of charges is slow enough, then the negative third moments will be measured experimentally through event-by-event analyses. Once such signals are measured, they serve direct evidence that the peak structure of corresponding susceptibility exists in the phase diagram of QCD, and that the matter on the far side of the phase transition, i.e. the quark-gluon plasma is created. The combination of the third moments of different channels, and their comparison with the numerical results in lattice QCD will bring various information on the phase structure and initial states created in heavy ion collisions at different energies.

\section{Focussing Effect and Antiproton-to-proton Ratio}

Ideas for experimental signatures for the presence of the critical point have mostly focused on fluctuations in certain observables related to the order parameter of the chiral transition [3, 15]. Unfortunately, several reasons throw doubt on the usefulness of fluctuation observables as practical signatures of the QCD critical point as explained in the previous section.

Here we propose a possible signature of the presence of a critical point in the QCD phase diagram, which may be more robust than fluctuations associated with the order parameter of the chiral phase transition. Our idea is based on the observation that the critical point serves as an attractor of the hydrodynamical trajectories in the $\mu_{B}-T$ plane describing the expansion of the hot matter [9]. We describe below how this focusing effect manifests itself in an experimental observable.

The universality argument tells us that the critical exponents around second order phase transitions are determined only by the dimensionality and symmetry of the system. The QCD critical point, if it exists, belongs to the same universality class as the 3-dimensional Ising model and liquid-gas phase transition [15]. The singular part of the thermodynamic variables near the critical point is a function of two variables, which can be mapped onto the variables characterizing the phase diagram of the 3-dimensional Ising model: the reduced temperature $r=\left(T-T_{c}\right) / T_{c}$ and the external magnetic field $h$. In the QCD phase diagram, the axis corresponding to $r$ points in the direction of the phase boundary; the direction of the axis associated with the variable $h$ is not known [8, 10]. However, it is clear that the critical region is more elongated along the $r$-direction, because the critical exponent associated with $r$ is larger than that associated with $h[8]$.

The focusing effect can now be understood as follows. The entropy density $s$ and the baryon density $n_{b}$ depend in different ways on $r$ and $h$. As a result, the ratio $s / n_{b}$, which is constant 
along an isentropic trajectory, assumes many different values in the vicinity of the critical point. Therefore, hydrodynamic trajectories for a range of different values of $s / n_{b}$ pass near the critical point, thus causing the focusing effect.

The extent of the focusing region depends on the size of the critical region in the $\mu_{B}-T$ plane, in which thermodynamic susceptibilities are significantly enhanced by the critical exponents. The size of the attractive basin can, in principle, be determined by lattice QCD simulations. At the moment this information is not available, as the location and even the existence of the critical point in QCD are not established. Model studies in simplified theories suggest that the size of the attractive region is sensitive to calculational details [16]. Here we will simply assume that the critical region is sufficiently large to induce a significant focusing effect. We use the model of Nonaka and Asakawa [9] to describe the influence of the critical point on the thermodynamic variables. In this model the entropy density is obtained by interpolation between the entropy densities of the hadronic and quark phase. Here we differ from ref. [9] by choosing the parameters $\Delta T_{\text {crit }}=20 \mathrm{MeV}$, $\Delta \mu_{\text {crit }}=100 \mathrm{MeV}$, and $D=0.5$.

The main characteristic of the fireball evolution in the presence of a critical point is that hydrodynamical trajectories, which would normally tilt to the right after crossing the phase boundary (see solid line in Fig. 3 for a smooth crossover or the dash-dotted line for a first order transition), make a detour into the vicinity of the critical point and then turn to the left as the temperature falls below $T_{c}$ (see dashed line in Fig. 3). For our argument, the important difference is the behavior just below $T_{c}$, where both $T$ and $\mu_{B}$ decrease for the trajectory through the critical point, while $\mu_{B}$ stays roughly constant or increases with falling temperature for trajectories away from the critical point. This difference can have visible consequences if hadron emission occurs over a finite range of temperatures, and if emission from different points along the trajectory can be discriminated. For instance, the ratio $\mu_{B} / T$ monotonically increases below $T_{c}$ along the "normal" (solid or dashdotted) trajectories in Fig. 3 , implying a falling antiproton-to-proton $(\bar{p} / p)$ ratio. On the other hand, the dashed trajectory in Fig. 3 implies an approximately constant or even slightly decreasing value of $\mu_{B} / T$ and thus a rising $\bar{p} / p$ ratio as the temperature falls below $T_{c}$.

In order to confirm this qualitative argument we present a quantitative analysis based on the assumption that the attractive basin of the critical point is reached in central $\mathrm{Pb}+\mathrm{Pb}$ collisions

at $40 \mathrm{GeV} / A$. In Fig. 4 we show the $\bar{p} / p$ ratio along the three trajectories shown in Fig. 4 as a function of the entropy density between $T_{c}$ and the chemical freezeout point, which has been determined to lie at $\left(\mu_{\mathrm{ch}}, T_{\mathrm{ch}}\right) \approx(400,145) \mathrm{MeV}$ by a statistical model fit to experimental data. As anticipated, the $\bar{p} / p$ ratio falls or remains constant between the phase boundary and chemical freezeout for the "normal" trajectories (solid and dash-dotted lines), but rises for the trajectory deformed by the presence of the critical point (dashed line).

We next discuss how baryon emission from different points along the hydrodynamical trajectory may be distinguished. We first note that data from $\mathrm{Au}+\mathrm{Au}$ collisions at RHIC have been explained by the assumption that the emission of hadrons with intermediate transverse momentum ( $p_{\mathrm{T}} \sim 2-5 \mathrm{GeV} / \mathrm{c}$ ) occurs at the phase boundary by recombination of constituent quarks [17, 18]. Bulk freezeout of hadrons, on the other hand, occurs when the mean free path of hadrons becomes comparable to the size of the fireball. The mean free path relevant to transport properties generally grows with increasing hadron momentum. This implies that hadrons with large transverse momentum should freeze out earlier, on average, than hadrons with a small transverse momentum. In the 


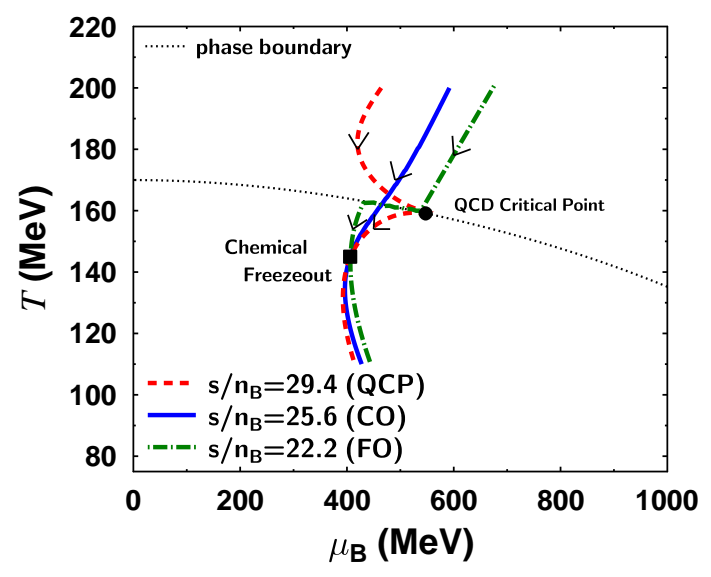

Figure 3: Hydrodynamical trajectories in the QCD phase diagram with and without the presence of a critical point. Possible trajectories in the $\mu_{B}-T$ plane in the absence of a critical point are shown as solid line (for a crossover transition) or dash-dotted line (for a first order transition); the trajectory in the presence of a critical point is shown as dashed line. All trajectories meet at the bulk chemical freezeout point. Arrows indicate the direction of time evolution.

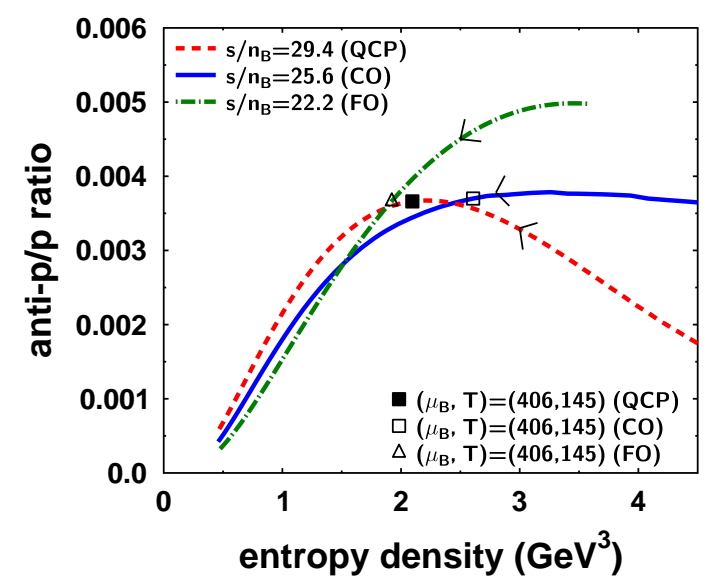

Figure 4: Antiproton-to-proton ratio along the trajectories shown in Fig.3 as a function of the entropy density. The curves start at the phase boundary $T_{c} \approx 160 \mathrm{MeV}$ and continue down to $T \approx 110 \mathrm{MeV}$. The location of the chemical freezeout point $\left(\mu_{\mathrm{ch}}, T_{\mathrm{ch}}\right)$ deduced from experimental data is indicated by the open and solid squares. Note that the $\bar{p} / p$ ratio only rises for the trajectory deformed by the critical point.

extreme, intermediate $p_{\mathrm{T}}$ hadrons may be produced at or near the phase boundary. This effect can also be understood by invoking detailed balance. A highly energetic hadron, impinging onto the fireball from the outside, would penetrate deeper into the matter than a low-energy hadron. Conversely, energetic hadrons will be emitted, on average from deeper inside the matter and thus earlier than low-energy hadrons.

The differential emission of baryons as a function of transverse momentum can be analyzed quantitatively in the framework of a microscopic hadron transport model, e.g. UrQMD [19, 20]. 

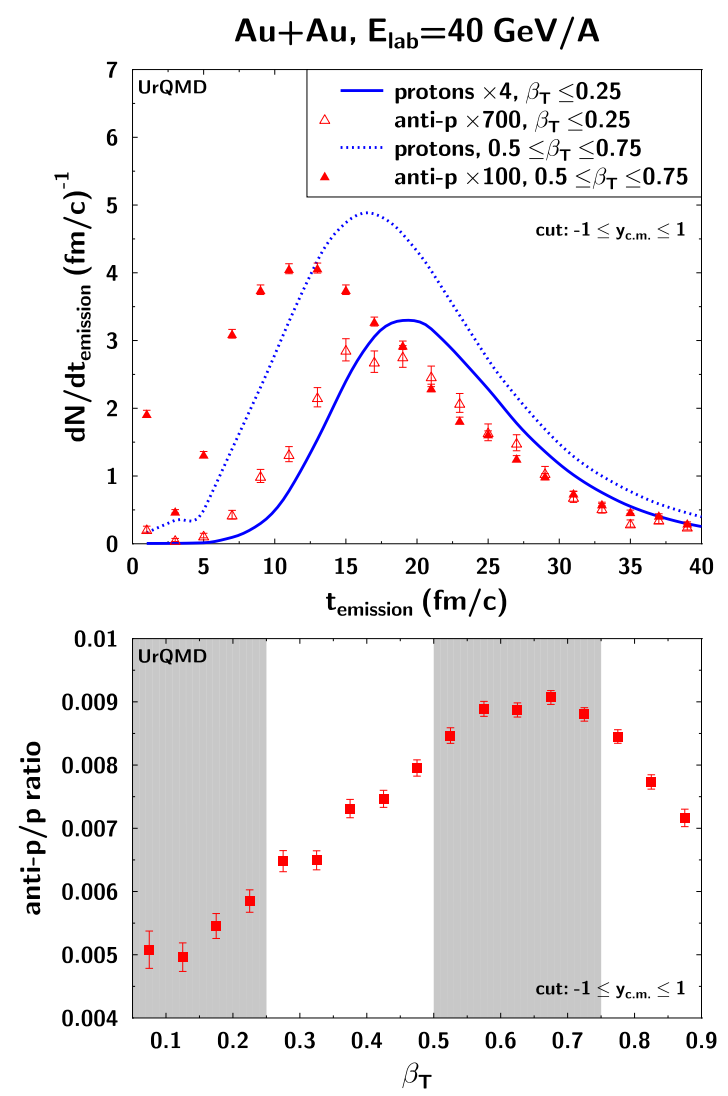

Figure 5: Top: UrQMD predictions for the emission time (last interaction time) distribution of protons and antiprotons in central $\mathrm{Au}+\mathrm{Au}$ collisions at $40 \mathrm{GeV} / \mathrm{A}$. The emission time distributions are shown separately for the kinematic windows $\beta_{\mathrm{T}}<0.25$ and $0.5<\beta_{\mathrm{T}}<0.75$. Fast particles are emitted on average significantly earlier than slow particles. Bottom: $\bar{p} / p$ ratio as a function of transverse velocity $\beta_{\mathrm{T}}$.

The bottom frame of Fig. 5 confirms the expectation from the solid line in Fig. 4 , that the $\bar{p} / p$ ratio should rise as a function of $\beta_{\mathrm{T}}$ in the absence of a critical point. Because the UrQMD calculation does not include finite-density corrections to the $p+\bar{p}$ annihilation cross section, the overall value of the ratio should not be compared with experiment. We also note that UrQMD does not contain any physics related to the QCD phase transition and only serves here as a model to study the correlation between emission time and transverse velocity. We expect the observed correlation to persist in the presence of a critical point. The fall in the $\bar{p} / p$ ratio for $\beta_{\mathrm{T}}>0.8$ is due to kinematical constraints on the $\bar{p}$ production and independent of collision size and energy. Since only a very small fraction of the baryon yield resides in the $\beta_{\mathrm{T}}>0.75$ range we have selected the $0.5<\beta_{\mathrm{T}}<0.75$ range as representative for high $\beta_{\mathrm{T}}$ and early freezeout nucleons.

In this section, we have shown that the evolution of the $\bar{p} / p$ ratio along isentropic curves between the phase boundary in the QCD phase diagram and the chemical freezeout point is strongly dependent on the presence or absence of a critical point. When a critical point exists, the isentropic trajectory approximately corresponding to hydrodynamical expansion is deformed, and the $\bar{p} / p$ ratio grows during the approach to chemical freezeout. If nucleons of high transverse momentum are chemically frozen out earlier than the slow nucleons, as it is suggested by microscopic simulations 
of hadronic dynamics, this result will translate into a $\bar{p} / p$ ratio that falls with increasing transverse momentum instead of a rise or flat behavior in scenarios without critical point. This behavior would only occur at those beam energies, for which the fireball reaches the critical point. Depending on the actual size of the attractive region around the critical point, the search for an anomaly in the $y_{\mathrm{T}}$ dependence of the $\bar{p} / p$ ratio may require small beam energy steps. Note that the location of the critical point in our model study was chosen such that it is encountered by the hydrodynamical trajectory for conditions reached for a beam energy of $40 \mathrm{GeV} / \mathrm{A}$ and a fixed-target. For a different location of the critical point, similar behavior would occur at other beam energies.

\section{References}

[1] T. Hatsuda, J. Phys. G 34 (2007) S287.

[2] M. A. Stephanov, PoS LAT2006 (2006) 024.

[3] M. A. Stephanov, K. Rajagopal, and E. V. Shuryak, Phys. Rev. Lett. 81 (1998) 4816; Phys. Rev. D 60 (1999) 114028.

[4] M. Asakawa, U. W. Heinz, and B. Muller, Phys. Rev. Lett. 85 (2000) 2072.

[5] S. Jeon and V. Koch, Phys. Rev. Lett. 85 (2000) 2076.

[6] Y. Hatta and M. A. Stephanov, Phys. Rev. Lett. 91 (2003) 102003 [Erratum-ibid. 91 (2003) 129901].

[7] S. Ejiri, F. Karsch, and K. Redlich, Phys. Lett. B633 (2006) 275; M. A. Stephanov, Phys. Rev. Lett. 102 (2009) 032301.

[8] B. Berdnikov and K. Rajagopal, Phys. Rev. D 61 (2000) 105017.

[9] C. Nonaka and M. Asakawa, Phys. Rev. C 71 (2005) 044904.

[10] Y. Hatta and T. Ikeda, Phys. Rev. C 67 (2003) 014028.

[11] C. Sasaki, B. Friman, and K. Redlich, Phys. Rev. D 75 (2007) 054026.

[12] T. Hatsuda and T. Kunihiro, Phys. Rept. 247 (1994) 221.

[13] T. Kunihiro, Phys. Lett. B271 (1991) 395.

[14] M. Asakawa and K. Yazaki, Nucl. Phys. A504 (1989) 668.

[15] M. A. Stephanov, K. Rajagopal, and E. V. Shuryak, Phys. Rev. D 60 (1999)114028.

[16] B.-J. Schaefer and J. Wambach, Phys. Rev. D75 (2007) 085015.

[17] R. J. Fries, B. Müller, C. Nonaka, and S. A. Bass, Phys. Rev. Lett. 90 (2003) 202303.

[18] V. Greco, C. M. Ko and P. Levai, Phys. Rev. Lett. 90 (2003) 202302.

[19] S. A. Bass et al., Prog. Part. Nucl. Phys. 41 (1998) 255.

[20] M. Bleicher et al., J. Phys. G 25 (1999) 1859. 